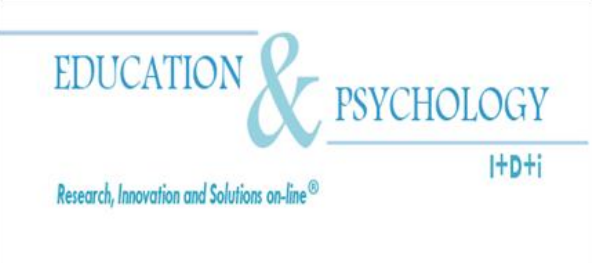

\title{
Cuestionario de Autoconcepto Físico (CAF) en una muestra de estudiantes chilenos
}

\section{Leandro Navas Martínez ${ }^{1}$, José Antonio Soriano Llorca ${ }^{1}$ y Francisco Pablo Holgado Tello ${ }^{2}$}

\author{
${ }^{1}$ Departamento de Psicología Evolutiva y Didáctica, Universidad de Alicante, \\ Alicante \\ ${ }^{2}$ Departamento de Metodología de las Ciencias del Comportamiento, Universi- \\ dad Nacional de Educación a Distancia, Madrid
}

\section{España}




\section{Resumen}

Introducción. El objetivo de este estudio es comprobar si se mantiene la estructura de seis dimensiones del Cuestionario de Autoconcepto Físico y valorar sus cualidades psicométricas con alumnado chileno.

Método. Han participado 1767 estudiantes, $45.8 \%$ chicos y $54.2 \%$ chicas, con una edad media de 18.39 años, procedentes de las regiones centro y sur de Chile. Se han realizado análisis factoriales confirmatorios, de fiabilidad y de discriminación.

Resultados. Los resultados obtenidos muestran que el modelo teórico se ajusta adecuadamente a los datos y que se reproduce la estructura factorial propuesta, siendo los factores fiables, lo que es una prueba de la validez de constructo del cuestionario.

Discusión y Conclusiones El Cuestionario de Autoconcepto Físico se muestra útil para la evaluación del autoconcepto físico del alumnado de Chile. Además, parece que existe una estructura de orden superior con factores de segundo orden que darían cuenta de los de primer orden.

Palabras Clave: autoconcepto físico, habilidad física, fuerza, atractivo físico, condición física 


\title{
The CAF Physical Self-Concept Questionnaire in a sample of Chilean students
}

\begin{abstract}
Introduction. The aim of this study was to verify if there is kept the structure of 6 dimensions of Physical Self-Concept Questionnaire and assess its psychometric qualities with Chilean students.
\end{abstract}

Method. One thousand seven hundred sixty-seven students took part in this research, $45.8 \%$ boys and $54.2 \%$ girls, with an average age of 18.39 years, from Central and South areas of Chile. Confirmatory factor, reliability and discrimination analyzes were conducted.

Results. The results obtained showed that the theoretical model fits the data adequately and that the structure of the six factors is reproduced, and these reliable, which, at the same time, provides further evidence of the validity of the questionnaire model.

Discussion and Conclusions. The Physical Self-Concept Questionnaire shown useful for the assessment of physical self-concept of students in Chile. Furthermore, it appears that there is a higher order structure with second-order factors that would account for the first order factors.

Keywords: physical self-concept, physical ability, strenght, physical attractiveness, physical condition

Reception: 05/27/13 Initial acceptance: 07/1413 Final acceptance: 11/06/13 


\section{Introducción}

La psicología, como ciencia que estudia el comportamiento, siempre ha otorgado atención al autoconcepto como variable de interés para explicar la conducta de los seres humanos. El autoconcepto se refiere a las nociones y opiniones que las personas tienen de sí mismas y se muestra como un buen indicador de la salud psicológica (Esnaola, Goñi y Madariaga, 2008) y como un gran predictor del rendimiento académico (Castejón y Miñano, 2010; Miñano y Castejón, 2011). Bien es cierto que, inicialmente, los resultados de las investigaciones realizadas muestran contradicciones porque se parte de una noción global y unidimensional del autoconcepto (Burns, 1979; Fitts, 1972). A partir de la publicación del trabajo de Shavelson, Hubner y Stanton (1976), se asume una noción multidimensional y jerárquica del autoconcepto. Según diversos autores (Castejón y Miñano, 2010; González-Pienda, Núñez, González-Pumariega y García, 1997; Sampascual, 2007) es multidimensional porque las diferentes autopercepciones se organizan en dimensiones específicas y tiene una estructura jerárquica porque las dimensiones más generales se subdividen en dominios más específicos tales como el social, el académico, el familiar, el personal o el físico.

Este estudio se centra en el autoconcepto físico que, aunque tradicionalmente se ha considerado un componente importante del autoconcepto (Esnaola et al., 2008; Esnaola y Revuelta, 2009), no ha sido estudiando como tal hasta finales de los años ochenta del siglo pasado (Fox, 1988; Fox y Corbin, 1989). El autoconcepto físico está configurado por las ideas, creencias o percepciones que se tienen en el ámbito físico sobre la propia habilidad, la fuerza, el atractivo, la condición física, la competencia deportiva, etc. (Esnaola y Revuelta, 2009). Dos modelos sobre la naturaleza multidimensional del autoconcepto físico pueden ser destacados. Por una parte, el modelo de cuatro subdominios de Fox y Corbin (1989) que establece que la autoevaluación física se subdivide en la competencia deportiva, el atractivo del cuerpo, la condición física y la fuerza física. Por otro lado, el modelo de Marsh, Richards, Johnson, Roche y Redmayne (1994) quienes señalan que la autopercepción física presenta nueve integrantes: actividad física, coordinación, grasa corporal, salud, competencia deportiva, fuerza, apariencia física, resistencia y flexibilidad. No obstante, existen otras dimensionalizaciones tales como la de Franzoi y Shields (1984), quienes distinguen tres dimensiones (habilidad física, apariencia física y conductas de control de peso), la de Bracken (1992) que establece cuatro dimensiones (competencia física, apariencia física, forma física y salud) o la de Sonstroem (1984) que aisla diversos componentes (estatura, peso, constitución, atractivo, fuerza, 
etc.). La estructura más aceptada para el autoconcepto físico, como dimensión del autoconcepto, es la propuesta por Fox y Corbin (1989) que considera las dimensiones competencia deportiva, condición física, atractivo físico y fuerza (Asci, Asci y Zorba, 1999; Goñi y Ruiz de Azúa, 2009).

El autoconcepto físico se relaciona con el riesgo de sufrir trastornos alimentarios (Rodríguez y Esnaola, 2009), con el bienestar psicológico, la actividad física y los estilos de vida saludable (Rodríguez, Goñi y Ruiz de Azúa, 2006; Reigal, Videra, Parra y Juárez, 2012), con las expectativas de éxito, el valor percibido y la dificultad percibida de la actividad física (Esnaola y Revuelta, 2009), con la práctica deportiva (Contreras, Fernández, García, Palou y Ponseti, 2010; Reigal, Videra, Márquez y Parra, 2013), con las influencias mediáticas (Ruiz de Azúa, Rodríguez y Goñi, 2005), con la actividad físico deportiva, la motivación y la satisfacción con la vida (Goñi e Infante, 2010; León, Núñez, Domínguez y Martín-Albo, 2012) y, por otra parte, se ha estudiado a lo largo del ciclo vital (Esnaola, 2008; Goñi, Rodríguez y Esnaola, 2010; Molero, Zagalaz-Sánchez y Cachón-Zagalaz, 2013), mostrando capacidad para establecer diferencias en función de la edad, del género, del índice de masa corporal o de la actividad deportiva (Goñi, Ruiz de Azúa y Rodríguez, 2004; Rodríguez et al., 2006).

En lo concerniente a la evaluación del autoconcepto físico, además de los cuestionarios para evaluar el autoconcepto en general que contienen una subescala para valorar el autoconcepto físico, como la Tennessee Self Concept Scale (Fitts, 1965), la Piers-Harris Children's Self Concept Scale (Piers, 1976) o el AF5 (García y Musitu, 1999), específicamente, para evaluar el autoconcepto físico, las escalas y cuestionarios más utilizados son la Pyhsical Self- Concept Scale, de Richards (1988), el Physical Self-Perception Profile (PSPP) de Fox y Corbin (1989), el Physical Self-Description Questionnaire (PSDQ) de Marsh et al. (1994), la versión reducida de éste (PSDQ-S) de Marsh, Martin y Jackson (2010), y el Children and Youth, Physical Self-Perception Profile (CY-PSPP) de Whitehead, (1995).

En lengua española, uno de los instrumentos más empleados ha sido el Cuestionario de Autoconcepto Físico (CAF) de Goñi, Ruiz de Azúa y Rodríguez (2006). Este cuestionario surgió para superar las limitaciones técnicas y los inconvenientes que mostró la traducción del CY-PSPP (Goñi et al., 2006) y ha sido aplicado en diferentes contextos (Fernández, Contreras, García y González, 2010; Infante y Goñi, 2009; Goñi e Infante, 2010; Molero, Ortega, Valiente y Zagalaz, 2010; Revuelta y Esnaola, 2011; Soriano, Navas y Holgado, 2011), mos- 
trando altos niveles de fiabilidad y de validez con estudiantes desde secundaria a universidad (Goñi et al., 2006) o con adultos mayores (Goñi et al., 2010) y, además de evaluar las cuatro dimensiones del autoconcepto físico, evalúa el autoconcepto general y el autoconcepto físico general. Para su elaboración se parte del modelo jerárquico y multidiemensional de Fox y Corbin (1989) en el que se establecen tres niveles. En el nivel más elevado está el autoconcepto general, en el nivel intermedio está el autoconcepto físico general y en el nivel más bajo los diferentes subdominios del autoconcepto físico (condición física, competencia deportiva, atractivo físico y fuerza). Si bien, los autores del CAF sustituyen el nombre de competencia deportiva por habilidad física.

Así, el CAF consta de las siguientes subescalas (Goñi et al., 2010; Goñi y Ruiz de Azúa, 2009; Rodríguez et al., 2006; Ruiz de Azúa, 2009): 1) Habilidad física (percepción de habilidades para la práctica de deportes, capacidad para apender deportes, seguridad personal para el deporte y predisposición ante los deportes); 2) Condición física (condición y forma física, energía y resistencia física, confianza en el estado físico); 3) Atractivo físico (autopercepción de la propia apariencia física, seguridad y satisfacción por la propia imagen); 4) Fuerza (sentirse fuerte, con capacidad para levantar peso, con seguridad ante ejercicios que demandan fuerza y predisposición para realizar tales ejercicios); 5) Autoconcepto físico general (sensaciones positivas -felicidad, orgullo, satisfacción, confianza- en lo físico), y 6) Autoconcepto general (satisfacción con uno mismo y con la vida en general).

En Chile se han realizado estudios con la Tennessee Self Concept Scale (Fitts, 1965, adaptada por Atallat, 1990; como, por ejemplo, Denegri, Opazo y Martínez, 2007), con la Piers-Harris Children's Self Concept Scale (Piers, 1976, adaptada por Gorostegui, 1992; véase Villarroel, 2002), con el AF5 (García y Musitu, 1999, adaptada por Véliz, 2010; véanse los trabajos de Droguett, 2011; Riquelme y Riquelme, 2011; Véliz y Apodaca, 2012a, 2012b) o con el Physical Self-Description Questionnaire Short-form (Marsh et al., 2010; como, por ejemplo, Espinoza, Rodríguez, Gálvez, Vargas y Yáñez, 2011). Sin embargo, pocas son las investigaciones realizadas con el CAF de Goñi et al. (2006) que presenta la ventaja de no tener que ser traducido.

\section{Objetivo}

Las normas para el desarrollo de estudios instrumentales, así como para la adaptación de los tests de una realidad cultural a otra están establecidas (Carretero-Dios y Pérez, 2005; 
Muñiz, Elosua y Hambleton, 2013) y señalan que, para cumplir las directrices de confirmación, es necesario realizar el análisis estadístico de los ítems y estudiar la dimensionalidad del instrumento. Por ello, la finalidad de este estudio es analizar las propiedades psicométricas y la equivalencia de la estructura factorial del CAF (Goñi et al., 2006) con alumnado chileno. En consecuencia, el objetivo de este trabajo es comprobar si la estructura factorial planteada por Goñi y Ruiz de Azúa (2009) para el CAF, se obtiene en una muestra de estudiantes chilenos, así como analizar sus propiedades psicométricas para determinar si resulta ser un instrumento fiable y válido con el alumnado de Chile.

\section{Método}

\section{Participantes}

Participan 1787 estudiantes (45.8\% hombres y 54.2\% mujeres) con edades comprendidas entre los 11 y los 50 años $(M=18.39 ; D T=4.44)$. De ellos, el 14.6\% cursa Enseñanza General Básica, el 34.5\% estudia Enseñanza Media, el 47.2\% realiza estudios universitarios y el 3.7\% restante recibe Formación Técnica. Todos provienen de diferentes localidades comprendidas entre las regiones del centro y sur de Chile (el 15\% de la Región Metropolitana de Santiago, el 3\% de la Región de Coquimbo, el 13\% del Región de Valparaíso, el 1.3\% de la Región del Libertador General Bernardo O’Higgins, el 16\% de la Región del Maule, el 42\% de la Región del Bío Bío, el 1\% de la Región de la Araucanía, el 7.7\% de la Región de Los Lagos y el 1\% de la Región de Magallanes) y se seleccionan por muestreo incidental. El tamaño de la muestra, según Arkin y Colton (1962), garantiza un error muestral inferior a $\pm 3 \%$ con un nivel de confianza del 95\% $(p=.5)$.

\section{Instrumento}

Se emplea el Cuestionario de Autoconcepto Físico (CAF) de Goñi et al. (2006). Está compuesto de 36 ítems que se valoran en una escala tipo Likert de 5 puntos en la que 1 significa falso y 5 verdadero.

1) La dimensión Habilidad Física está compuesta por los ítems 1, 6, 17, 23, 28 y 33, que expresan ideas como "No tengo cualidades para los deportes" o "Me veo torpe en las actividades deportivas" y representa la percepción de la propia habilidad para practicar deporte $(\alpha=.84)$. 
2) La dimensión Condición Física $(\alpha=.88)$ está integrada por los ítems $2,7,11,18$, 24 y 29, que expresan ideas como "Tengo mucha energía física" o "Puedo correr y hacer ejercicio durante mucho tiempo sin cansarme" y que se relaciona con la confianza en el propio estado físico y en la autopercepción de resistencia para llevar a cabo actividades físicas intensas.

3) La dimensión Atractivo Físico agrupa a los ítems 8, 12, 19, 25, 30 y 34, con expresiones como "Me cuesta tener un buen aspecto físico" o "Siento confianza en cuanto a la imagen física que transmito", que hace referencia a la percepción del propio aspecto físico y al grado de satisfacción que se tiene con la imagen que se ofrece a los demás $(\alpha=.87)$.

4) La dimensión Fuerza ( $\alpha=.83$ ) está formada por los elementos 3, 9, 13, 20, 31 y 35 , que expresan ideas como "Soy capaz de realizar actividades que exigen fuerza" o "Soy fuerte" y se relaciona con la percepción de fortaleza y de capacidad para llevar a cabo actividades que la precisan (como, por ejemplo, levantar peso).

5) La dimensión Autoconcepto Físico General está integrada por las cuestiones 4, 14, 16, 21, 26 y 36, con enunciados como "Físicamente me siento peor que los demás" o "Físicamente me siento bien", y se refiere a percepciones positivas relativas al físico tales como satisfacción, confianza, orgullo, etc. $(\alpha=.86)$.

6) Finalmente, la subescala Autoconcepto General $(\alpha=.84)$ agrupa a los elementos 5, 10, 15, 22, 27 y 32, que expresan ideas como "Me siento feliz" o "Desearía ser diferente", y evalúa el nivel en el que el sujeto está satisfecho con él mismo y con la vida en términos generales.

En los ítems indirectos $(5,6,7,8,9,15,16,22,23,24,25,26,32,33,35$ y 36) se invierte la escala. El coeficiente de fiabilidad para el total del cuestionario oscila entre .73 y .92 (Ruiz de Azúa, 2009).

\section{Procedimiento}

Tras requerir el consentimiento informado no retribuido de los participantes (o de las familias del alumnado en el caso de los menores de edad), siguiendo los criterios de Bioética de la Comisión Nacional de Investigación Científica y Tecnológica de Chile (Lira, 2008), el 
cuestionario se administra en el aula habitual. Se cumplimenta en grupo, pero asegurando que no se comenten las respuestas entre compañeros, de forma voluntaria y se instruye a los sujetos cómo deben responder siguiendo las instrucciones de Goñi et al. (2006), se les solicita la máxima sinceridad y se les garantiza el anonimato y la confidencialidad de las respuestas. Algunos estudiantes son excluidos del estudio por tener respuestas en blanco o por no tener el consentimiento informado de los progenitores. Así, los participantes son finalmente 1760.

\section{Análisis estadísticos}

El diseño es correlacional básico. Se llevan a cabo análisis descriptivos, análisis factorial confirmatorio (AFC) de la estructura propuesta por Goñi et al. (2006), análisis de fiabilidad y un análisis factorial de segundo orden.

\section{Resultados}

En la Tabla 1 se resumen los resultados de los análisis descriptivos (véanse las cuatro primeras columnas). Los valores de asimetría y curtosis se encuentran dentro del rango \pm 1.96, por lo que se infiere que las variables se ajustan razonablemente a una distribución normal.

Tabla 1. Análisis descriptivos y de fiabilidad de los elementos del CAF

\begin{tabular}{lcccccc}
\hline Variable & $M$ & $D T$ & $A$ & $C$ & $\alpha$ & $r_{\text {i-total }}$ \\
\hline Item 1 & 3.38 & 1.22 & -0.42 & -0.60 & .93 & .61 \\
Item 2 & 3.16 & 1.15 & -0.19 & -0.66 & .93 & .62 \\
Item 3 & 2.67 & 1.15 & 0.11 & -0.72 & .93 & .45 \\
Item 4 & 3.63 & 1.18 & -0.54 & -0.53 & .93 & .64 \\
Item 5 & 3.69 & 1.29 & -0.60 & -0.76 & .93 & .30 \\
Item 6 & 3.82 & 1.26 & -0.69 & -0.66 & .93 & .43 \\
Item 7 & 3.58 & 1.27 & -0.45 & -0.87 & .93 & .48 \\
Item 8 & 3.39 & 1.33 & -0.31 & -1.03 & .93 & .46 \\
Item 9 & 3.47 & 1.26 & -0.36 & -0.86 & .93 & .32 \\
Item 10 & 4.23 & 1.01 & -1.38 & 1.48 & .93 & .50 \\
Item 11 & 3.38 & 1.23 & -0.38 & -0.71 & .93 & .68 \\
Item 12 & 3.57 & 1.20 & -0.53 & -0.56 & .93 & .65 \\
Item 13 & 3.68 & 1.16 & -0.55 & -0.49 & .93 & .52 \\
Item 14 & 3.53 & 1.21 & -0.46 & -0.65 & .93 & .69 \\
Item 15 & 4.18 & 1.19 & -1.29 & 0.57 & .93 & .38 \\
Item 16 & 3.93 & 1.27 & -0.89 & -0.38 & .93 & .55 \\
Item 17 & 2.73 & 1.24 & 0.14 & -0.89 & .93 & .54 \\
Item 18 & 2.88 & 1.27 & 0.03 & -0.95 & .93 & .60 \\
\hline
\end{tabular}




\begin{tabular}{lllllll}
\hline Item 19 & 3.44 & 1.15 & -0.35 & -0.59 & .93 & .64 \\
Item 20 & 2.76 & 1.29 & 0.13 & -1.05 & .93 & .55 \\
Item 21 & 3.66 & 1.10 & -0.52 & -0.36 & .93 & .62 \\
Item 22 & 3.64 & 1.45 & -0.60 & -1.05 & .93 & .55 \\
Item 23 & 3.81 & 1.32 & -0.76 & -0.65 & .94 & .28 \\
Item 24 & 3.41 & 1.30 & -0.33 & -0.93 & .93 & .47 \\
Item 25 & 3.84 & 1.31 & -0.78 & -0.58 & .93 & .62 \\
Item 26 & 3.75 & 1.30 & -0.67 & -0.70 & .93 & .60 \\
Item 27 & 3.77 & 1.05 & -0.66 & 0.04 & .93 & .52 \\
Item 28 & 3.46 & 1.26 & -0.41 & -0.81 & .93 & .61 \\
Item 29 & 3.49 & 1.16 & -0.36 & -0.60 & .93 & .67 \\
Item 30 & 3.61 & 1.16 & -0.57 & -0.32 & .93 & .42 \\
Item 31 & 3.25 & 1.21 & -0.28 & -0.74 & .93 & .54 \\
Item 32 & 3.95 & 1.24 & -0.87 & -0.43 & .94 & .21 \\
Item 33 & 3.88 & 1.24 & -0.79 & -0.47 & .93 & .54 \\
Item 34 & 3.66 & 1.18 & -0.63 & -0.36 & .93 & .46 \\
Item 35 & 3.21 & 1.36 & -0.15 & -1.11 & .93 & .37 \\
Item 36 & 3.92 & 1.26 & -0.84 & -0.46 & .93 & .60 \\
\hline
\end{tabular}

El modelo teórico de las distintas dimensiones del CAF (se ha descrito en la subsección Instrumento), se somete a un AFC, utilizando mínimos cuadrados no ponderados robusto. En la Tabla 2 se muestra la solución completamente estandarizada.

Tabla 2. Solución completamente estandarizada del modelo de Goñi et al. (2006). En la parte inferior los índices de fiabilidad y discriminación de cada una de las dimensiones

\begin{tabular}{ccccccc}
\hline Items & $\begin{array}{c}\text { Habilidad } \\
\text { física }\end{array}$ & $\begin{array}{c}\text { Condición } \\
\text { física }\end{array}$ & $\begin{array}{c}\text { Atractivo } \\
\text { Físico }\end{array}$ & $\begin{array}{c}\text { Fuerza } \\
\text { Autoconcepto } \\
\text { físico general }\end{array}$ & $\begin{array}{c}\text { Autoconcepto } \\
\text { general }\end{array}$ \\
\hline 1 & .82 & -- & -- & -- & -- & -- \\
2 & -- & .77 & -- & -- & -- & -- \\
3 & -- & -- & -- & .70 & -- & -- \\
4 & -- & -- & -- & -- & .75 & -- \\
5 & -- & -- & -- & -- & -- & .44 \\
6 & .57 & -- & -- & -- & -- & -- \\
7 & -- & .58 & -- & -- & -- & -- \\
8 & -- & -- & .58 & -- & -- & - \\
9 & -- & -- & -- & -46 & -- & -- \\
10 & -- & -- & -- & -- & -- & .73 \\
11 & -- & .80 & -- & -- & -- & -- \\
12 & -- & -- & -85 & -- & -- & -- \\
13 & -- & -- & -- & .80 & -- & - \\
14 & -- & -- & -- & -- & .81 & - \\
15 & -- & -- & -- & -- & -- & .56 \\
16 & -- & -- & -- & -- & .68 & -- \\
17 & .73 & -- & -- & -- & -- & - \\
18 & -- & .75 & -- & -- & -- & - \\
19 & -- & -- & .81 & -- & -- & - \\
20 & -- & -- & -- & .85 & -- & - \\
\hline
\end{tabular}




\begin{tabular}{ccccccc}
\hline 21 & -- & -- & -- & -- & .74 & -- \\
22 & -- & -- & -- & -- & -- & .76 \\
23 & .39 & -- & -- & -- & -- & -- \\
24 & -- & .57 & -- & -- & -- & -- \\
25 & -- & -- & .82 & -- & -- & -- \\
26 & -- & -- & -- & -- & .73 & -- \\
27 & -- & -- & -- & -- & -- & .69 \\
28 & .82 & -- & -- & -- & -- & -- \\
29 & -- & .83 & -- & -- & -- & -- \\
30 & -- & -- & .54 & -- & -- & -- \\
31 & -- & -- & -- & .79 & -- & -- \\
32 & -- & -- & -- & -- & -- & .30 \\
33 & .71 & -- & -- & -- & -- & -- \\
34 & -- & -- & .63 & -- & -- & - \\
35 & -- & -- & -- & .53 & -- & - \\
36 & -- & -- & -- & -- & .71 & -- \\
\hline$\alpha$ & .78 & .83 & .82 & .80 & .84 & .68 \\
$r_{i-\text { total }}$ & .54 & .61 & .59 & .57 & .61 & .42 \\
\hline
\end{tabular}

Se obtienen los siguientes índices globales de bondad de ajuste: $\chi^{2}$ Satorra-Bentler $=$ $3546.26(g l=579 ; p<.05) ;$ REMSEA $=.08 ; \mathrm{GFI}=.98 ; \mathrm{AGFI}=.97 ; \mathrm{CFI}=.96 ; \mathrm{NNFI}=.95 \mathrm{y}$ $\mathrm{RMR}=.08$. Estos resultados permiten concluir que el modelo tiene un adecuado ajuste, teórico y empírico, a los datos por lo que no es necesario añadir o modificar parámetros en él.

Tras realizar los análisis de fiabilidad (coeficiente de consistencia interna alfa de Cronbach) y de discriminación (coeficiente de correlación $r$ de Pearson entre la puntuación del elemento y la del total del cuestionario sin incluir la de ese elemento), el total del cuestionario presenta un $\alpha=.94$ y un índice medio de discriminación de .52. En la Tabla 1 (véanse las dos últimas columnas) se ofrecen los valores para cada elemento. Los índices de consistencia interna y de discriminación media de cada uno de los factores son también aceptables (véase la fila inferior de la Tabla 2).

En la Tabla 3 se ofrece la matriz de correlaciones bivariadas entre los distintos factores. Todos los valores de los coeficientes $r$ de Pearson obtenidos son estadísticamente significativos $(p<.01)$. Se ve que las cuatro primeras variables están muy relacionadas entre sí y que hay una fuerte relación entre las dos últimas. Esto, junto con la interpretación teórica, indica que habilidad física, atractivo físico, condición física y fuerza hacen referencia a subdominios específicos del autoconcepto físico, mientras que autoconcepto físico general y autoconcepto general son variables que se encuentran en un nivel jerárquico y de concreción 
superior (Goñi et al., 2006; Ruíz de Azúa, 2009) y ello permite plantear un segundo modelo teórico (Modelo 2) compuesto por dos factores de segundo orden. Uno de ellos, el Factor 1, explicaría las cuatro primeras variables y el Factor 2 las dos últimas.

Tabla 3. Coeficientes de correlación entre los factores del CAF

\begin{tabular}{|c|c|c|c|c|c|c|}
\hline & $\mathrm{HF}$ & $\mathrm{CF}$ & $\mathrm{AF}$ & FUE & AUTOFIS & AUTOGEN \\
\hline $\mathrm{HF}$ & 1 & & & & & \\
\hline $\mathrm{CF}$ & .90 & 1 & & & & \\
\hline $\mathrm{AF}$ & .51 & .65 & 1 & & & \\
\hline FUE & .77 & .75 & .42 & 1 & & \\
\hline AUTOFIS & .65 & .77 & .99 & .53 & 1 & \\
\hline AUTOGEN & .52 & .58 & .90 & .38 & .92 & 1 \\
\hline
\end{tabular}

La solución completamente estandarizada del Modelo 2, se muestra en la Figura 1 en la que se representa el modelo estructural del planteamiento teórico examinado.

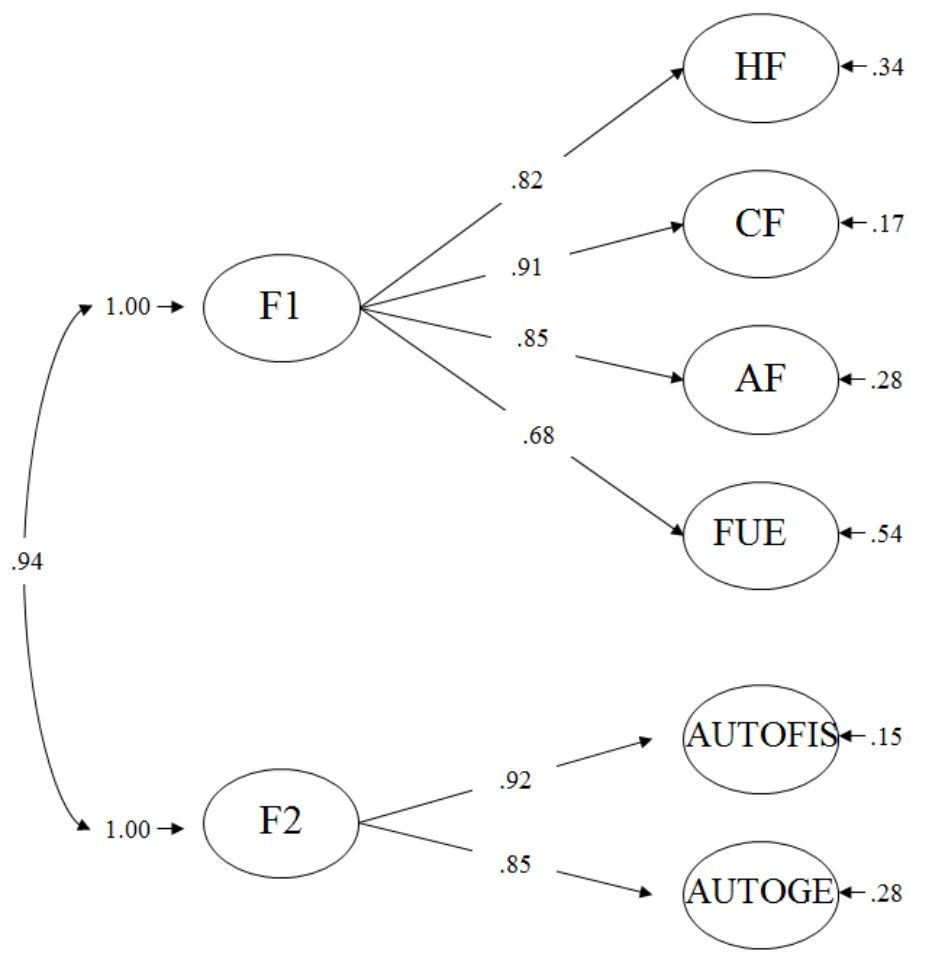

Figura 1. Solución estandarizada del modelo estructural del Modelo 2

Nota $: \mathrm{HF}=\quad$ Habilidad física $; \mathrm{CF}=$ Condición física; $\mathrm{AF}=$ Atractivo físico; FUE = Fuerza; AUTOFIS = Autoconcepto físico general; AUTOGE = Autoconcepto general; F1 = Factor 1; $\mathrm{F} 2=$ Factor 2 
Además, se calculan el ECVI y el CAIC que se usan para medir el ajuste comparativo entre dos o más modelos (los valores cada vez más pequeños indican un mejor ajuste), suponen una medida de la parsimonia del modelo al considerar el número de parámetros e indican el grado en que el modelo es reproducible en otras muestras (Bandalos, 1993). En la Tabla 4, se aprecia que el Modelo 2 también representa los datos adecuadamente, que hay un incremento significativo en $\chi^{2}$ y que los valores de ECVI y de CAIC muestran que el Modelo 1 es más parsimonioso y replicable. No obstante, los valores de los otros índices de ajuste justifican el uso del Modelo 2.

Tabla 4. Índices de ajuste de los modelos 1 y 2

\begin{tabular}{llllllllll}
\hline Modelo & RMSEA & GFI & AGFI & ECVI & CAIC & $\chi^{2}$ & $G l$ & $\Delta \chi^{2}$ & $\Delta g l$ \\
\hline 1 & .08 & .98 & .97 & 4.66 & 4214.82 & $3546.2^{*}$ & 579 & --- & --- \\
2 & .08 & .93 & .92 & 6.26 & 5446.27 & $4846.8^{*}$ & 588 & 1301 & 9 \\
\hline
\end{tabular}

Nota: $*(\mathrm{p}$-valor $=.000)$

\section{Discusión y conclusiones}

El objetivo de este estudio era analizar la estructura factorial del CAF (Goñi et al., 2006) y sus cualidades psicométricas con estudiantes chilenos. El modelo teórico sometido a prueba se compone de las seis dimensiones propuestas por los autores del cuestionario. Los resultados obtenidos en el AFC permiten concluir que el modelo presenta un adecuado ajuste a los datos y no es necesario modificar o incorporar parámetros en el mismo. Ello viene a indicar que la estructura factorial obtenida con participantes españoles (Fernández et al., 2010; Infante y Goñi, 2009; Goñi e Infante, 2010; Goñi et al., 2006; Molero et al., 2010; Revuelta y Esnaola, 2011; Soriano et al., 2011) se reproduce con estudiantes chilenos y, además se ve confirmada la estructura tetradimensional y jerárquica del autoconcepto físico planteada por Fox y Corbin (1989), lo que viene a coincidir con los estudios llevados a cabo por diversos investigadores (Asci et al., 1999; Goñi et al., 2006; Hayes, Crocker y Kowalsky, 1995; Holgado, Soriano y Navas, 2009; Kosmidou, Proios y Giannitsopoulou, 2013; Kudláček, Kudláček, Kudláček y Kudláček, 2010; Kumartasli, 2013; Lindwall y Jonson, 2001; Moreno, 1997; Page, Ashford, Fox y Biddle, 1993; Schlegel y Fialova, 2013) en diferentes países (Turquía, Canadá, España, República Checa, Suecia, Grecia o Gran Bretaña). También es 
cierto que hay estudios en los que del AFC resultan tres dimensiones (por ejemplo, Atienza, Balaguer, Moreno y Fox, 2004). Hay que tener en cuenta que la mayor parte de los trabajos recién mencionados se realizan con el PSPP y que el CAF se diferencia de ese instrumento de evaluación en “a) la delimitación conceptual de las cuatro dimensiones; b) el cambio de denominación de competencia deportiva por habilidad física para una de las dimensiones; c) la inclusión de nuevos ítems; y d) el cambio de formato en la redacción de los ítems" (Goñi et al., 2010, p. 461).

Además, los resultados de este trabajo ofrecen apoyo empírico, aparte de a la estructura factorial obtenida por Goñi et al. (2006), a la validez de constructo del CAF. Se confirma que el modelo teórico ajusta a las respuestas del cuestionario, con lo que se puede deducir que la diferenciación entre la habilidad física, la condición física, el atractivo físico y la fuerza dan cuenta de las autopercepciones físicas de los adolescentes, jóvenes y personas de la edad adulta temprana de Chile.

Por otra parte, los resultados del análisis de fiabilidad permiten deducir que, con estudiantes chilenos, los factores del CAF son fiables y, salvo en el caso del autoconcepto general, no hay muchas diferencias entre los coeficientes obtenidos en este estudio y aquellos de los que informa Ruíz de Azúa (2009). Incluso, para el total del cuestionario, el alfa obtenido es ligeramente superior al que aporta Goñi (2009) en la ficha técnica del instrumento. Ello indica que el CAF es igualmente fiable con alumnado de Chile.

Una de las cuestiones más relevantes de este trabajo ha sido la propuesta del segundo modelo, que aporta otras dos nuevas variables cuyo interés para futuras investigaciones pasa por estudiar su relación con la estructura jerárquica del autoconcepto físico. Concretamente, el Factor 1 hace referencia a que las personas, dentro de su autopercepción física global, establecen subdominios diferenciados que se encuentran en un nivel de concreción más bajo y en un rango jerárquico inferior (las subdimensiones de la parte baja de la jerarquía); mientras que el Factor 2 va referido a autopercepciones más abstractas, más generales y en un rango jerárquico superior (las dimensiones de la parte alta del modelo jerárquico), tal como ha sido defendido por Fox y Corbin (1989), Goñi et al. (2006) y Goñi y Ruíz de Azúa (2009). Esta dimensionalización de segundo orden es un aspecto a considerar en investigaciones futuras, dadas las relaciones que el autoconcepto físico presenta con la satisfacción vital y la percepción de salud (Videra-García y Reigal- Garrido, 2013). 
Ejerciendo la autocrítica, tres limitaciones están presentes en este trabajo. La primera es que los participantes proceden sólo de las regiones del centro y del sur del país, lo que puede suponer una amenaza para la posibilidad de generalizar estos resultados. Ampliar la muestra en aquellas regiones poco representadas o no consideradas en esta investigación es un área de trabajo de cara al futuro. La segunda limitación proviene del propio instrumento de evaluación, que se basa en el autoinforme y que puede contener los sesgos que se derivan de la deseabilidad social. Y la tercera es que hay niveles educativos que están infrarrepresentados y que deberían ampliarse en estudios subsecuentes.

La principal aplicación práctica que se deriva de este estudio es que se puede emplear el CAF como un cuestionario más para la evaluación psicopedagógica de estudiantes chilenos, pues es un instrumento que cumple los requisitos de validez de constructo y de fiabilidad. Bien es cierto que para obtener evidencias de la validez externa quedan pendientes de realizarse estudios en los que se analicen la validez de criterio (relaciones entre el test y un criterio que lo prediga), la validez convergente (relaciones entre el test y otros test que evalúen lo mismo) y la validez discriminante (relaciones entre el test y otras variables de las que debe diferenciarse) con muestras de estudiantes chilenos. Esta es una tarea que, por alejarse de los objetivos de este trabajo, queda pendiente para futuras investigaciones.

Como conclusiones destacar que: 1) el modelo tetradimensional y jerárquico del autoconcepto físico se ve confirmado; 2) se obtiene apoyo empírico para la estructura factorial lograda por los autores del CAF; 3) se obtienen índices de fiabilidad y de discriminación adecuados, a pesar de las diferencias contextuales y socioculturales; 4) se obtienen evidencias de la validez de constructo del CAF; 5) se hallan dos nuevas variables (F1 y F2) que se relacionan con la estructura jerárquica del autoconcepto físico, y 6) hay que seguir investigando con participantes chilenos para soslayar las limitaciones que presenta este trabajo y para valorar la validez externa del CAF en Chile. 


\section{Referencias}

Arkin, H. y Colton, R. (1962). Tables for statiscians. Nueva York, NY: Barnes \& Noble.

Asci, F. H., Asci, A. y Zorba, E. (1999). Cross-cultural validity and reliability of Physical Self-Perception Profile. International Journal of Sport Psychology, 30, 399-406.

Atallat, R. (1990). Validación de la Escala de Autoconcepto de Tennessee en estudiantes de Enseñanza Media de Teтисо (Tesis para el título de Psicólogo). Universidad de la Frontera, Temuco, Chile.

Atienza, F. L., Balaguer, I., Moreno, Y. y Fox, K. R. (2004). El perfil de autopercepción física: propiedades psicométricas de la versión española y análisis de la estructura jerárquica de las autopercepciones físicas. Psicothema, 16(3), 461-467.

Bandalos, D. L. (1993). Factor influencing cross-validation of confirmatory factor analysis models. Multivariate Behavioral Research, 28, 351-374. doi: http://dx.doi.org/10.1207/s15327906mbr2803_3

Bracken, B. A. (1992). Multidimensional Self-Concept Scale. Austin, TX: Pro-Ed.

Burns, R. B. (1979). The self-concept. Theory, measurement, development and behavior. Nueva York, NY: Longman.

Carretero-Dios, H. y Pérez, C. (2005). Normas para el desarrollo y revisión de estudios instrumentales. International Journal of Clinical and Health Psychology, 5(3), 521-551.

Castejón, J. L. y Miñano, P. (2010). Motivación y autoconcepto en contextos escolares. En J. L. Castejón, C. González, R. Gilar y P. Miñano (Eds.), Psicología de la Educación (pp. 201-256). Alicante, España: ECU.

Contreras, O. R., Fernández, J. G., García, L. M., Palou, P. y Ponseti, J. (2010). El autoconcepto físico y su relación con la práctica deportiva en estudiantes adolescentes. Revista de Psicología del Deporte, 19(1), 23-39.

Denegri, M., Opazo, C. y Martínez, G. (2007). Aprendizaje cooperativo y desarrollo del autoconcepto en estudiantes chilenos. Revista de Pedagogía, 28(81), 13-41.

Droguett, L. (2011). Rasgos psicológicos asociados al ajuste social y personal del alumnado adolescente (Tesis doctoral). Recuperada de http://www.ehu.es/argitalpenak/images/stories/tesis/Ciencias_Sociales/DROGUETT\% 20LATORRE.pdf 
Esnaola, I. (2008). El autoconcepto físico durante el ciclo vital. Anales de Psicología, 24(1), $1-8$.

Esnaola, I., Goñi, A. y Madariaga, J. M. (2008). El autoconcepto: perspectivas de investigación. Revista de Psicodidáctica, 13(1), 69-96.

Esnaola, I. y Revuelta, L. (2009). Relaciones entre la actividad física, autoconcepto físico, expectativas, valor percibido y dificultad percibida. Acción Psicológica, 6(2), 31-43. doi: http://dx.doi.org/10.5944/ap.6.2.219

Espinoza, O. L., Rodríguez, R. F., Gálvez, C. J., Vargas, C. P. y Yáñez, S. R. (2011). Valoración del autoconcepto físico en estudiantes universitarios y su relación con la práctica deportiva. Motricidad Humana, 12(1), 22-26. Recuperado de http://dialnet.unirioja.es/descarga/articulo/3706586.pdf

Fernández, J. G., Contreras, O. R., García, L. M. y González, S. (2010). Autoconcepto físico según la actividad físico-deportiva realizada y la motivación hacia ésta. Revista Latinoamericana de Psicología, 42(2), 251-263.

Fitts, W. H. (1965). Manual for Tennessee Self-Concept Scale. Los Ángeles, CA: Western Psychological Services.

Fitts, W. H. (1972). The self-concept and behavior: Overview and supplement. Tennesse, TN: Nashville.

Fox, K. R. (1988). The self-esteem complex and youth fitness. Quest, 40(3), 230-246. doi: http://dx.doi.org/10.1080/00336297.1988.10483903

Fox, K. R. y Corbin, C. B. (1989). The Physical Self-Perception Profile: Development and preliminary validation. Journal of Sports and Exercise Psychology, 11, 408-430.

Franzoi, S. L. y Shields, S. A. (1984).The Body Esteem Sacale, multidimensional structure and sex differences in a college population. Journal of Personality Assesment, 48(2), 173-178.

García, F. y Musitu, G. (1999). AF5, Autoconcepto Forma 5. Madrid, España: TEA.

González-Pienda, J. A., Núñez, J. C., González-Pumariega, S. y García, M. (1997). Autoconcepto, autoestima y aprendizaje escolar. Psicothema, 9(2), 271-289.

Goñi, A. (2009) (Coord.). El autoconcepto físico. Madrid: Pirámide. 
Goñi, E. e Infante, G. (2010). Actividad físico-deportiva, autoconcepto físico y satisfacción con la vida. European Journal of Education and Psychology, 3(2), 199-208.

Goñi, A., Rodríguez, A. y Esnaola, I. (2010). Las autopercepciones físicas en la edad adulta y en la vejez. Psicothema, 22(3), 460-467.

Goñi, A. y Ruiz de Azúa, S. (2009). La estructura interna del autoconcepto físico. En A. Goñi (Coord.), El autoconcepto físico (pp. 81-97). Madrid: Pirámide.

Goñi, A., Ruiz de Azúa, S. y Rodríguez, A. (2004). Deporte y autoconcepto físico en la preadolescencia. Apunts. Educación Física y Deportes, 77, 18-24.

Goñi, A., Ruiz de Azúa, S. y Rodríguez, A. (2006). Cuestionario del Autoconcepto Físico. Manual. Madrid: EOS.

Gorostegui, M. E. (1992). Adaptación y construcción de las normas para la escala de autoconcepto para niños de Piers-Harris (Tesis para el título de Psicólogo). Escuela de Psicología, Pontificia Universidad Católica, Santiago de Chile.

Hayes, S. D., Crocker, P. R. y Kowalski, K. (1995). Gender differences in physical selfperceptions, global self-esteem and physical activity. Evaluation of physical selfperceptions profile model. Journal of Sport Behavior, 22(1), 1-14.

Holgado, F. P., Soriano, J. A. y Navas, L. (2009). El Cuestionario de Autoconcepto Físico (CAF): Análisis factorial confirmatorio y predictivo sobre el rendimiento académico global y específico del área de Educación Física. Acción Psicológica, 6(2), 93-102. doi: http://dx.doi.org/10.5944/ap.6.2.224

Infante, G. y Goñi, E. (2009). Actividad físico-deportiva y autoconcepto físico en la edad adulta. Revista de Psicodidáctica, 14(1), 49-61. doi: http://dx.doi.org/10.1387/RevPsicodidact.250

Kosmidou, E., Proios, M. y Giannitsopoulou, E. (2013). Physical self worth, athletic engagement and goal orientations in greek female athletes. Pamukkale Journal of Sport Sciences, 4(2), 79-93.

Kudláček, M., Kudláček, M., Kudláček, V. y Kudláček, V. (2010). Components/factors of the Czech version of the physical self-perception profile (PSPP-CZ) among high school students. Acta Universitatis Palackianae Olomucencis Gymnica, 40(4), 25-30. 
Kumartasli, M. (2013). Investigation of body attractiveness in adult males and sedentary males who are interested in body building. Life Science Journal, 10(3), 796-799.

León, J., Núñez, J. L., Domínguez, E. y Martín-Albo, J. (2012). Motivación intrínseca, autoconcepto físico y satisfacción con la vida en practicantes de ejercicio físico: Análisis de un modelo de ecuaciones estructurales en el entorno de programación R. Revista Iberoamericana de Psicología del Ejercicio y el Deporte, 8(1), 39-58.

Lindwall, M. y Johnson, U. (2001). Social physique anxiety and its relationship to physical self-perceptions and perceived body discrepancy. En A. Papaioannuo, M. Goudas e Y. Theodorakis (Eds.), Proceedings of the 10th World Congress of Sport Psychology, 1 (pp. 223-224). Skiatos, Greece: Christodoulidi.

Lira, E. (Ed.). (2008). Bioética en Investigación en Ciencias Sociales. 3er Taller organizado por el Comité Asesor de Bioética de FONDECYT-CONICYT. Santiago de Chile: CONICYT. Recuperado de http://www.conicyt.cl/fondecyt/files/2012/10/Libro-3Bio\%C3\%A9tica-en-investigaci\%C3\%B3n-en-ciencias-sociales.pdf

Marsh, H. W., Martin, A. J. y Jackson, S. (2010). Introducing a short version of the Physical Self Description Questionnaire: New strategies, Short-form evaluative criteria, and applications of factor analyses. Journal of Sport and Exercise Psychology, 32, 438482.

Marsh, H. W., Richards, G. E., Johnson, S., Roche, L. y Tremayne, P. (1994). Physical SelfDescription Questionnaire: Psychometric properties and a multitrait-multimethod analysis of relation to existing instruments. Journal of Sport and Exercise Psychology, $16,270-305$

Miñano, P. y Castejón, J. L. (2011). Variables cognitivas y motivacionales en el rendimiento académico en Lengua y Matemáticas: un modelo estructural. Revista de Psicodidácti$c a, 16(2), 203-230$.

Molero, D., Ortega, F., Valiente, I. y Zagalaz, M. L. (2010). Estudio comparativo del autoconcepto físico en adolescentes en función del género y del nivel de actividad físicodeportiva. Retos: Nuevas Tendencias en Educación Física, Deporte y Recreación, 17, $38-41$. 
Molero, D., Zagalaz-Sánchez, M. L. y Cachón-Zagalaz, J. (2013). Estudio comparativo del autoconcepto físico a lo largo del ciclo vital. Revista de Psicología del Deporte, 22(1), 135-142.

Moreno, Y. (1997). Propiedades Psicométricas del Perfil de Autopercepción Física (PSPP). (Tesis doctoral). Universidad de Valencia. Valencia, España.

Muñiz, J., Elosua, P. y Hambleton, R. K. (2013). Directrices para la traducción y adaptación de los tests: segunda edición. Psicothema, 25(2), 151-157. doi: http://dx.doi.org/10.7334/psicothema2013.24

Page, A., Ashford, B., Fox, K. R. y Biddle, S. (1993). Evidence of cross-cultural validity for the Physical Self-Perceptions Profile. Personal Individual Differences, 1(4), 585-590. doi: http://dx.doi.org/10.1016/0191-8869(93)90151-R

Piers, E. (1976). Manual for the Piers-Harris Children's Self Concept Scale. Los Ángeles, CA: Couseling Recorder and Test.

Reigal, R., Videra, A., Márquez, M. V. y Parra, J. L. (2013). Autoconcepto físico multidimensional y barreras para la práctica física en la adolescencia. Apunts. Educación Física y Deportes, 111(1), 23-28.

Reigal, R., Videra, A., Parra, J. L. y Juárez, R. (2012). Actividad físico deportiva, autoconcepto físico y bienestar psicológico en la adolescencia. Retos. Nuevas Tendencias en Educación Física, Deporte y Recreación, 22, 19-23.

Revuelta, L. y Esnaola, I. (2011). Clima familiar deportivo y autoconcepto físico en la adolescencia. European Journal of Education and Psychology, 4(1), 19-31.

Richards, G. E. (1988). Physical Self-Concept Scale. Sydney: Australian Outwand Bound Foundation.

Riquelme, E. y Riquelme, P. (2011). Análisis psicométrico confirmatorio de la medida multidimensional del test de Autoconcepto forma 5 en español (AF5), en estudiantes universitarios de Chile. Psicologia, Saúde \& Doenças, 12(1), 91-103. Recuperado de http://www.scielo.gpeari.mctes.pt/pdf/psd/v12n1/v12n1a06.pdf

Rodríguez, A. y Esnaola, I. (2009). Los trastornos de la conducta alimentaria y el autoconcepto físico. En A. Goñi (Coord.), El autoconcepto físico (pp. 173-192). Madrid, España: Pirámide. 
Rodríguez, A., Goñi, A. y Ruiz de Azúa, S. (2006). Autoconcepto físico y estilos de vida en la adolescencia. Intervención Psicosocial, 15(1), 81-94. doi: http://dx.doi.org/10.4321/S1132-05592006000100006

Ruiz de Azúa, S. (2009). La medida del autoconcepto físico. En A. Goñi (Coord.), El autoconcepto físico (pp. 99-110). Madrid: Pirámide.

Ruíz de Azúa, S., Rodríguez, A. y Goñi, A. (2005). Variables socioculturales en la construcción del autoconcepto físico. Cultura y Educación, 17(3), 225-238. doi: http://dx.doi.org/10.1174/113564005774462582

Sampascual, G. (2007). Psicología de la educación. Madrid, España: UNED.

Schlegel, P. y Fialova, L. (2013). Physical self in Czech and Swedish male fitness center visitors. Česká kinantropologie, 17(3), 75-81.

Shavelson, R. J., Hubner, J. J. y Stanton, J. C. (1976). Self concept: Validation of construct interpretattions. Review of Educational Research, 46(3), 407-441. doi: http://dx.doi.org/10.3102/00346543046003407

Soriano, J. A., Navas, L. y Holgado, F. P. (2011). El autoconcepto físico y su relación con el género y la edad en estudiantes de educación física. Apunts. Educación Física y Deportes, 106(4), 36-41.

Sonstroem, R. J. (1984). Exercise and self-esteem. Exercise and Sport Sciences Review, 12, 123-155. doi: http://dx.doi.org/10.1249/00003677-198401000-00007

Véliz, A. L. (2010). Dimensiones del autoconcepto en estudiantes chilenos: un estudio psi$\begin{array}{llll}\text { cométrico } & \text { (Tesis de }\end{array}$ https://www.educacion.gob.es/teseo/imprimirFicheroTesis.do?fichero=19823

Véliz, A. y Apodaca, P. (2012a). Dimensiones del autoconcpeto en estudiantes chilenos : un estudio psicométrico. Revista Educativa Hekademos, 11, 47-58. Recuperado de http://dialnet.unirioja.es/descarga/articulo/4059766.pdf

Véliz, A. y Apodaca, P. (2012b). Niveles de autoconcepto, autoeficacia académica y bienestar psicológico en estudiantes universitarios de la ciudad de Temuco. Salud \& Sociedad, 3(2), 131-150. Recuperado de http://pepsic.bvsalud.org/pdf/salsoc/v3n2/v3n2a02.pdf 
Videra-García, A. y Reigal-Garrido, R. (2013). Autoconcepto físico, percepción de salud y satisfacción vital en una muestra de adolescentes. Anales de Psicología, 29(1), 141147. doi: http://dx.doi.org/10.6018/analesps.29.1.132401

Villarroel, V. (2002). Autoconcepto y rendimiento escolar. Lectura y Vida, 23(2). Recuperado de http://www.lecturayvida.fahce.unlp.edu.ar/numeros/a23n2/23_02_Villarroel.pdf

Whitehead, J. R. (1995). A study of children's self-perceptions using an adapted Physical Self-Perception Questionnaire. Pediatric Exercise Science, 7, 133-152. 